\title{
Database Design of Physics Experimental Equipment Management System
}

\author{
Xiang Teng \\ Bohai University, Jinzhou, P.R. China \\ yyq8369@qq.com
}

\begin{abstract}
Keywords: Physics experimental equipment; database structural design; conceptual structural design; logical structural design; physical design
\end{abstract}

\begin{abstract}
Physics is an experimental inquiry-based discipline, rational and effective preparation and management of experimental equipment, is a necessary prerequisite for completing the exploration of experiments, but in the real life, it is the effectiveness of experimental equipment management that is concerning, and database design as an foundation for experimental equipment management system is very important, this paper mainly studies the database design of physics experimental equipment management system. Firstly, studies the conceptual structural design in database design, it is the key to database design; secondly, describes the logical structural design, specifically in the form of a table; finally, studies the physical structural design including the design content and design principles. Database design will directly affect the efficiency and achievement of physics experimental equipment management system, so the database design of physics experimental equipment management system studied in this paper is significance.
\end{abstract}

\section{Introduction}

Physics is both a basic discipline and is an experimental science, its development and innovation are closely linked with physical experiments, physical experiments is an important part in the process of physics researching, and is an important means of developing students' practical ability, creativity, the ability to integrate theory with practice, etc., experimental equipment is the security of completing experiment and vital auxiliary means, but the management of experimental equipment is a tedious work [1], so the experimental equipment management system came into being, and for equipment management, the most basic design is the database design, reasonable database design of physics experimental equipment management system can continually improve the quality of physical experiment learning, for the healthy development of experiment and practice has a positive significance. This paper studies the database design methods and procedures, which focuses on conceptual structural design, logical structural design and physical structural design of physics experimental equipment management system database.

\section{Conceptual Structural Design}

Conceptual design is also known as conceptual structural design, according to synthesizing, inducting and abstracting the user needs to form a specific DBMS-independent conceptual model, it can be expressed by the E-R diagram, with the characters easy to understand and change [2].

E-R diagram is an entity relationship diagram, it means that using the form of figure to represent the links among the entities, it is a description of the conceptual model in the real world, mainly consists of three parts, namely the entity set, attributes and links, in which the entity set is represented by a rectangular box, the entity refers to the object that objectively exists and mutually distinguish, it is both specific object and abstract concept. The entity in this system mainly includes administer, teachers, experimental equipment, equipment category and so on; attribute describes a characteristic value of an entity is represented by an oval with undirected edges to connect it with the corresponding entity type, in the data table the attribute is field, in this system, the attributes of teachers entity are TeacherID, TeacherName, TeacherSex, E-mail and others, the attributes of experimental equipment entity are EquipID, EquipName, EquipPrice, EquipFormat, EquipModel, 
EquipStatus, EquipNum, Producer and so on, the attributes of administrator entity are AdminID, AdminName, AdminSex, AdminPassword, AdminRights and so on, the attributes of using equipment entity are UsingID, EquipID, UsingTime, UsingNum and others, the attributes of equipment category entity are CategoryID, CategoryName, CategoryRights and Remarks; the link represents the connection between sets of two or more entities, represented by the diamond and using undirected edges to connect together with a related entity type, meanwhile signed the type of link beside the undirected edges, the type of link includes one-to-one $(1: 1)$, one-to-many $(1: n)$ and many to many (m : $\mathrm{n}$ ) three types [3].

In the database of the physics experimental equipment management system studied in this paper, it includes administrator information table, teachers information table, experimental equipment information table, equipment category information table, using equipment information table and others table structures, each table structure includes many fields, and each field includes many restrains, such as major key, foreign key and others, in this system between the administrator and management system is many-to-many $(\mathrm{m}: \mathrm{n})$; between the teachers and experimental equipment is many-to-many ( $\mathrm{m}: \mathrm{n}$ ); between the using equipment and experimental equipment is many-to-many; between the equipment category and experimental equipment is many-to-many ( $\mathrm{m}: \mathrm{n}$ ); between the management system and experimental equipment is one-to-many $(1: n)$. In this paper, the conceptual structural design is represented in form of E-R diagram, the conceptual model structure diagram is shown in Fig. 1.

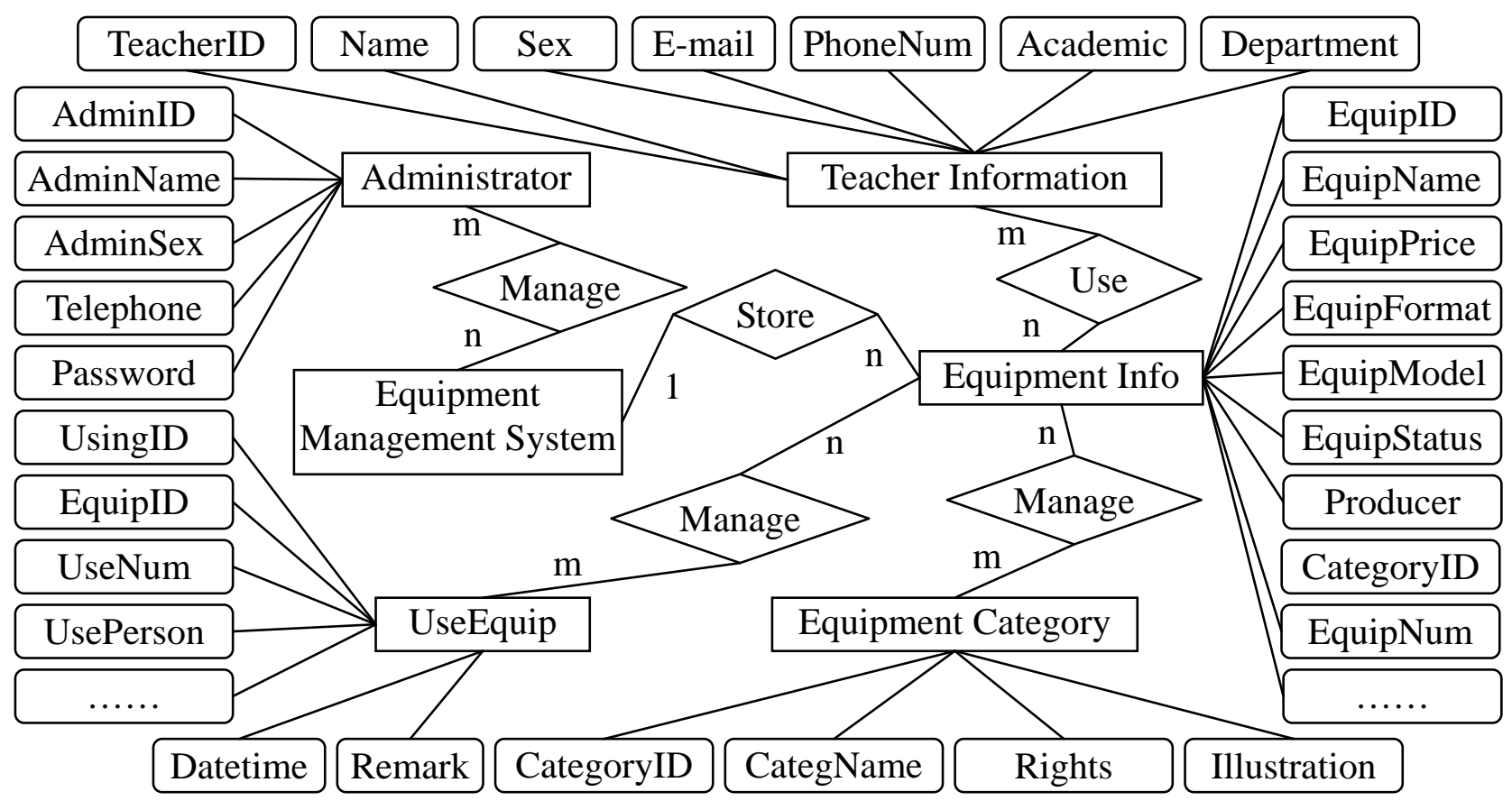

Fig. 1. E-R diagram of physics experimental equipment management system

\section{Logical Structural Design}

Design steps of logical structural design as follows, firstly, makes the conceptual structure convert into the general model, such as relationship, net and hierarchical model; secondly, makes the converted relationship, net, hierarchical model transform to the data model supported by a specific DBMS; and then according to requirements provided by user, on the basis of the basic table to create the outer mode formed the necessary view for data. The results of logical structural design is not single, so it has many logical structure, in order to enhance the performance of physics experimental equipment management database application system, it also need to appropriately modify the structure of data model and optimize data model, and then to advance the speed of querying data in the database. Due to the essence of logical structural design is converting E-R 
diagram in the process of conceptual structural design into the entity relationship diagrams, so it follows the principles as follows [4].

An entity type converts into a relational schema, the attribute and code of entity is the attribute and code of relationship; An m: $\mathrm{n}$ link converts into a relational schema, in which the code of relationship is a combination of an entity code; A 1: n link can be converted into a separate relational schema, may also correspond to the n-side relationship consolidation mode; A 1: 1 link can be converted into a separate relational schema and can also be combined with either end of the corresponding relational schema; A multi-link among three or more entities converts into a relational schema, each other of entities code connected with multi-link and the attributes of link itself convert into the attributes of contact; The link among entities in the same entity set, namely itself link, it can also treat in accordance with $1: 1,1: n$ and $m: n$ three cases above; The relational schema with the same code can be combined; The logical structural design results of physics experimental equipment management system database design is shown in Table 1.

Table 1. Logical structure of tables in database

\begin{tabular}{|c|c|c|c|c|c|c|c|}
\hline \multicolumn{4}{|c|}{ "Equipment Category" Table } & \multicolumn{4}{|c|}{ "Teacher Information" Table } \\
\hline 1 & CategoryID & varchar & 10 & 1 & TeacherID & varchar & 10 \\
\hline 2 & CategoryName & varchar & 50 & 2 & TeacherName & varchar & 20 \\
\hline 3 & Remarks & text & & 3 & TeacherSex & varchar & 2 \\
\hline \multicolumn{4}{|c|}{ "Administrator" Table } & 4 & E-mail & varchar & 50 \\
\hline 1 & AdministratorID & varchar & 10 & 5 & PhoneNum & varchar & 20 \\
\hline 2 & AdminName & varchar & 20 & 6 & Academics & varchar & 50 \\
\hline 3 & AdminPassword & varchar & 50 & 7 & Department & text & \\
\hline 4 & AdminSex & varchar & 2 & $\ldots$ & ...... & $\ldots \ldots$ & $\ldots \ldots$ \\
\hline 5 & TelephoneNum & varchar & 50 & \multicolumn{4}{|c|}{ "Equipment Information" Table } \\
\hline \multicolumn{4}{|c|}{ "Using Equipment" Table } & 1 & EquipID & varchar & 20 \\
\hline 1 & UsingID & varchar & 10 & 2 & EquipName & varchar & 50 \\
\hline 2 & EquipID & varchar & 20 & 3 & EquipPrice & varchar & 100 \\
\hline 3 & UsingNum & varchar & 100 & 4 & EquipFormat & varchar & 50 \\
\hline 4 & Remarks & text & & 5 & EquipModel & varchar & 50 \\
\hline 5 & UsingPerson & varchar & 100 & 6 & EquipStatus & text & \\
\hline 6 & UsingTime & datetime & 8 & 7 & CategoryID & varchar & 20 \\
\hline 7 & ReturnTime & datetime & 8 & $\ldots$ & $\ldots \ldots$ & $\ldots \ldots$ & $\ldots \ldots$ \\
\hline
\end{tabular}

\section{Physical Structural Design}

The first step of physical design is set up database, firstly build a Wlsyqc database in the SQL server database system, and then found tables in database. Database accessed method is a technology of quickly accessing data in database, generally DBMS can provide a lot of accessed methods, such as indexing method, clustering method and others [5]. In the physical design of physics experimental equipment management system the accessed method is indexing method, indexing method is according to application request to determine build index, combined index or single index for what attributes. For this system, the record in database can include hundred thousand, in order to search basic data in data tables, we can build index, in experimental equipment table, based on field "EquipID" to build index; in teachers information table, based on field "TeachersID" to build index; in using equipment table, based on field "UsingID" to build index; in administrator information table, based on field "AdminID" to build index; in equipment catagory table, based on field "CatagoryID" to build index. Clustering method is to enhance the querying speed of some attributes (or attributes combination); it is a method stored the tuple with same attributes value in continuous physical block [6]. Determine the storage structure of database, the storage structure refers to the physical structure, specially refers to the storage location and storage 
structure in the database internal mode for data, according to the actual application condition, in the database of system studied in this paper tables and built indexes are stored dividually, it can achieve the goal of enhancing the efficiency for physics reading and writing. Due to the data size requested physics experimental equipment management system is not very huge relatively, so the data is stored in a disk, but from the angle of security, we need to backup at fixed periods, due to data backup and log files backup are only used when the breakdown recovered and the data size is very huge, so it is stored in different disk from other database objects, such as table, index, etc.. In the stage of physical design also need to evaluate the physical structure of database, mainly refers to its time and space efficiency.

\section{Conclusion}

Physics is a basic discipline, its development and innovation are closely linked with the physical experiment, it can be said that any innovations in physics are derived from experiments. Therefore, the physical experiment should become an important part of creating students innovation [7], the experiment is an important part of physics research, by physical experiments, on the one hand to deepen the understanding and mastery of the physics theoretical knowledge, inspired the enthusiasm and interest of learning physics knowledge; on the other hand to meet the imagine of many theory linking practical idea proposed by students in the learning physics and creativity demand, to cultivate innovation and manipulative ability [8]. Experimental equipment as an important part of the experiment, its management work is very essential, and therefore experimental equipment management system came into being, for the purposes of management system, database design is particularly important. This paper studies the conceptual design, logical design and physical design in the physics experimental equipment management system database, and laid a good foundation for the design and development of physics experimental equipment management system even for physical experiments provide an important supporting role.

\section{Acknowledgement}

This work is supported by "Teaching Reform Project of Bohai University: Research and practice on modern physics virtual experiment simulation system of development.

\section{References}

[1] Z. B. Chen, W. Chen, "Design and Implementation of Laboratory Equipment Management System," Journal of Fuzhou University, vol. 36, no. 1, pp. 181, 2008.

[2] L. D. Ke, "Database Design and Analysis," Science \& Technology Information, vol. 5, no. 26, pp. 79, 2007.

[3] Baidu Baike, "namespace," http://baike.baidu.com/view/15236.htm?fr=aladdin, 2015-1-14.

[4] Z. Y. Duan, "Database Design Methodology," Journal of Nanchang College of Education, vol. 20, no. 4, pp. 85-86, 2005.

[5] S. Y. Cui, Y. Zhang, C. Zeng, H. J. Feng, X. C. Xing, "Database Physical Structure Optimization Technology," Journal of Software, vol. 24, no. 2, pp. 762-765, 2013.

[6] J. L. Lin, "The Design and Implementation of Scientific Research Management Information System's Database," Master's Degree of Beijing University of Chemical Technology.

[7] H. S. Ma, "Practice of Designing and Research Physics Experiments," Physics Experimentation, vol. 24, no. 11, pp. 29-30, 2004.

[8] F. Q. Zuo, "University Physics Experiment Teaching Reform," Technical Physics Teaching, vol. 17, no. 3, pp. 40-41, 2009. 